INPLASY

PROTOCOL

To cite: Li et al. Exercise effect on weight and body composition for breast cancer patients undergoing chemotherapy: A Metaanalysis. Inplasy protocol 202150016. doi:

10.37766/inplasy2021.5.0016

Received: 04 May 2021

Published: 04 May 2021

Corresponding author: Jinyu Zhang

zjy2001_4@163.com

Author Affiliation:

Shanghai University of Traditional Chinese Medicine

Support: Shanghai Health

Commission.

Review Stage at time of this submission: Data analysis.

Conflicts of interest: None declared.

\section{Exercise effect on weight and body composition for breast cancer patients undergoing chemotherapy: A Meta-analysis}

Li, X1; Wang, J2; Zhang, J3; Zhang, N4; Wu, C5; Geng, Z6; Zhou, J7; Dong, L8.

Review question / Objective: The research questions of the present study are, "Is exercise an effective way to prevent weight gain and harmful changes in body composition for breast cancer patients undergoing chemotherapy?" and, "Do the exercise interventions in previous studies show clear effectiveness in every kind of body composition?" This systematic review aims to analyze the current literature and assess the exercise effect for breast cancer patients undergoing chemotherapy in reducing weight and changing body composition by a meta-analysis of randomized controlled trials (RCTs).

Information sources: We searched the CINAHL, PubMed, Embase, and EBSCO to identify suitable articles published in English. We also searched SinoMed to find suitable studies in Chinese.

INPLASY registration number: This protocol was registered with the International Platform of Registered Systematic Review and Meta-Analysis Protocols (INPLASY) on 04 May 2021 and was last updated on 12 May 2021 (registration number INPLASY202150016).

\section{INTRODUCTION}

Review question / Objective: The research questions of the present study are, "Is exercise an effective way to prevent weight gain and harmful changes in body composition for breast cancer patients undergoing chemotherapy?" and, "Do the exercise interventions in previous studies show clear effectiveness in every kind of body composition?" This systematic review aims to analyze the current literature and assess the exercise effect for breast cancer patients undergoing chemotherapy 
in reducing weight and changing body composition by a meta-analysis of randomized controlled trials (RCTs).

Rationale: The effect of exercise on breast cancer patients during chemotherapy is not yet clear. We searched the existing literature and conducted a systematic review to identify the effect of exercise on weight and body composition for breast cancer patients undergoing chemotherapy.

Condition being studied: Breast cancer is a major threat to women's health in both developed and developing countries, being both the most common form of female cancer and associated with the highest cancer mortality rate. Chemotherapy is commonly used as an adjuvant treatment strategy for primary breast cancer and provides significant survival benefits, but it can cause weight gain and negative changes in body composition, which may impair the quality of life, treatment completion, and survival outcomes. In healthy people, physical exercise is the key to prevent weight gain and negative changes in body composition. However, the effect of exercise on breast cancer patients during chemotherapy is not yet clear. We searched the existing literature and conducted a systematic review to identify the effect of exercise on weight and body composition for breast cancer patients undergoing chemotherapy.

\section{METHODS}

Search strategy: We searched the CINAHL, PubMed, Embase, and EBSCO to identify suitable articles published in English. We also searched SinoMed to find suitable studies in Chinese. Searches did not have a year limitation and covered the period January 1974 - December 2020. Among the keywords and mesh terms used, the following were the most significant: breast neoplasms, breast, breast diseases, neoplasms, drug therapy, chemotherapy, neoadjuvant chemotherapy, chemical therapy, weight, BMI, body fat, lean mass, and BMD.
Participant or population: Breast cancer patients undergoing chemotherapy.

Intervention: Exercise.

Comparator: Usual care.

Study designs to be included: Randomized controlled trials (RCTs).

Eligibility criteria: We included RCTs that evaluated the effect of exercise on weight or body composition, where exercise included aerobic exercise, resistance exercise or a combination of several types. To be included in this systematic review, the exercise intervention needed to coincide with the chemotherapy.

Information sources: We searched the CINAHL, PubMed, Embase, and EBSCO to identify suitable articles published in English. We also searched SinoMed to find suitable studies in Chinese.

Main outcome(s): Weight; weight changes; BMI; body fat; lean mass; handgrip and BMD.

Data management: Two authors will independently extract data. Any disagreement will be resolved by discussion until consensus is reached or by consulting a third author. The following data will be extracted: author, year of publication, the country where the study was conducted, study period, original inclusion criteria, the total number of people included in the study, interventions, and time of training.

Quality assessment / Risk of bias analysis: Two reviewers will independently assess the quality of the selected studies according to the Cochrane collaboration's tool for randomized controlled trials. Items will be evaluated in three categories: Low risk of bias, unclear bias, and high risk of bias. The following characteristics will be evaluated: Random sequence generation (selection Bias) Allocation concealment (selection bias)Blinding of participants and personnel (performance bias) incomplete 
outcome data (attrition bias)Selective reporting (reporting bias).

Strategy of data synthesis: Reviewer Manager v5.3 (Review Manager (RevMan) [Computer program]. Version 5.3. Copenhagen The Nordic Cochrane Centre, 2014) was used to conduct all statistical analyses. In the summary statistics of the meta-analysis of the continuity results, we either used the standardized mean difference (SMD) or weighted mean difference (WMD). SMD with $95 \% \mathrm{Cl}$ was chosen. We encountered different assessment instruments used to measure the same outcome variable in the different studies (e.g., for body fat and lean mass outcomes). We used the $I^{2}$ statistic to evaluate statistical heterogeneity. The level of $\mathbf{I}^{2}$ was recommended by the Cochrane manual (12: $0 \%, 25 \%, 50 \%$ and $75 \%$ represent no, low, medium, and high heterogeneity). For outcome weight, some studies reported final values of body weight after intervention and some reported weight change across the intervention period. In our meta-analysis, we did not combine final values with change scores.

Subgroup analysis: We will consider subgroups such as clinic type, duration of intervention.

Sensitivity analysis: After excluding a lowquality study, the combined effect size was re-estimated and compared with the results of the Meta-analysis before exclusion to explore the extent of the effect of the study on the combined effect size and the robustness of the results. If the results did not change significantly after the exclusion, it indicates that the sensitivity is low and the results are more robust and credible; on the contrary, if large differences or even diametrically opposite conclusions are obtained after the exclusion, it indicates that the sensitivity is high and the robustness of the results is low, and great care should be taken when interpreting the results and drawing conclusions, suggesting the existence of important and potentially biased factors related to the effects of the intervention, and the source of the controversy needs to be further clarified.

\section{Country(ies) involved: China.}

Keywords: breast cancer, chemotherapy, weight, body composition, nursing, exercise, systematic review and metaanalysis.

\section{Contributions of each author:}

Author 1 - Xinyu Li.

Email: yolilxy0801@gmail.com

Author 2 - Jiaqi Wang.

Author 3 - Jinyu Zhang.

Email: zjy2001_4@163.com

Author 4 - Nan Zhang.

Author 5 - Caiqin Wu.

Author 6 - Zhaohui Geng.

Author 7 - Jie Zhou.

Author 8 - Lu Dong. 